and hospital informative system, medical practice-related issues (including section 12(2) approval and medical indemnity). The Trainees' Duty section briefed on time-tabling and clinical duty. The Specialty-specific Guide provided important information related to training. Lastly, the section of Health Board-related Information highlighted the administrative structure of the NHS Health Board, important contact numbers, link to information. Specialty specific sections were created for general adult psychiatry and old age psychiatry as there is no other higher training of psychiatry in North Wales at the moment. Further sections in the pipeline include substance misuse and liaison psychiatry. Conclusion. This induction manual is neither prescriptive nor exhaustive. It serves as a generic reference to facilitate new trainees in their adjustment process. Further review and revision will be conducted before every induction process to ensure the information is up-to-date and incorporating new input from the trainees.

\section{Audit of documentation of observations on mental health services for older people (MHSOP) wards following implementation of nervecentre}

Nitya Rathi

Northumbria NHS Foundation Trust

doi: 10.1192/bjo.2021.573

Aims. Nervecentre is an application that can be used on mobile devices and desktop computers to record and view physical observations amongst other tools. An audit had been done previously assessing the practice of recording observations using paper documentation. That audit had recommended the use of Nervecentre to improve the recording of observations. This audit was undertaken following the introduction of Nervecentre for documentation of physical observations. The aims were to evaluate if the transition to electronic documentation of NEWS (National Early Warning Score) observations on Nervecentre has improved practice in comparison to paper documentation and to evaluate if our practice could be improved by implementing electronic observations for psychiatric observations in addition.

Method. Data were collected over a 10-day period looking at all the documented observations from all inpatients on the MHSOP wards that met the inclusion criteria. Data were collected on the recording of psychiatric observations (recorded on paper charts) and physical observations (recorded on Nervecentre). The data were collated and analysed. The new data were compared to the original data from prior to the introduction of NerveCentre and the findings were presented at a local meeting.

Result. This audit has highlighted that the documentation of physical observations on MHSOP wards has greatly improved since Nervecentre was introduced. There was an improvement in recording of physical observations in almost all domains measured. NEWS scores were correctly documented $100 \%$ of the time compared to $87 \%$ previously. Raised NEWS scores were correctly escalated to a senior and reviewed $80 \%$ of the time compared to $0 \%$ previously. It has also highlighted that the quality of documentation regarding psychiatric observations could be improved as we are not currently meeting local or national guidance.

Conclusion. The most likely cause for the improvement in the recording of the physical observations is the implementation of Nervecentre. Nervecentre prompts users when observations are due, removes the risk of calculation errors and allows for observations to be directly escalated. Implementing Nervecentre for psychiatric observations may similarly improve the quality of these observations therefore improving patient safety.
A pilot to assess the feasibility and potential clinical utility of enhanced sleep management on inpatient wards in a mental health trust

Ambrina Roshi ${ }^{1 \star}$, Rose McGowan ${ }^{2}$, Lauren Roberts ${ }^{2}$, Stuart Watson ${ }^{3}$, Kirstie Anderson ${ }^{2}$, Patrick Keown ${ }^{3}$, Rod Bowles ${ }^{3}$, Ron Weddle $\mathrm{W}^{3}$ and Sophie Connolly ${ }^{3}$

${ }^{1}$ St Nicholas Hospital, Cumbria Northumberland Tyne Wear NHS Foundation Trust; ${ }^{2}$ Newcastle University and ${ }^{3}$ Cumbria, Northumberland, Tyne And Wear NHS Foundation Trust ${ }^{\star}$ Corresponding author.

doi: $10.1192 /$ bjo.2021.575

Aims. To assess the feasibility and utility of introducing the following changes on to in-patient units:

Structural and cultural adaptation to create a sleep friendly ward environment

A "Protected Sleep Time" between midnight and 6am

Routine screening for sleep disorders, including obstructive sleep apnoea and restless leg syndrome

Background. Insomnia and other sleep disturbances are cause, correlate and consequence of psychiatric disorders. Routine hourly night time observations, ward noise, bright lights at night time, sleep disorders, insufficient exercise, insufficient day light exposure, too much caffeine and inappropriate psychotropic use are all causes of disturbed sleep (Horne 2018).

Method. Seven wards participated in a pilot (SleepWell). These consisted of one male and two female Acute Wards (General Adult), a High Dependency Unit, a Neurorehabilitation ward, an in-patient dementia service and one rehabilitation ward. These wards were supported via an existing trust management structure and the pilot was specifically supported by two trust managers (RW and RB) and by a clinical director (PK). The expectation was that each ward would identify a sleep champion from existing staff to facilitate the changes. A "product" was developed which identified core sleep management features but, in addition, wards were not confined to these. The existing policy that all inpatients should be checked each hour over night was suspended for the pilot wards and the patients had protected sleep time (PST) if the MDT agreed that it was clinically appropriate.

Quantitative and qualitative techniques were used to identify facilitators of change, impact on sleep and, outcome.

Result. Protected sleep was viewed positively by all staff and approximately $50 \%$ of patients on the pilot wards were able to have PST at some point in their admission. Routine sleep disorder assessments were harder to implement and $33 \%$ of patients were screened. There were no deaths or significant events on patients due to PST. Hypnotic use on the pilot wards reduced. It is anticipated that PST where it is safe will be rolled out across all adult and old age wards in the trust.

Conclusion. With support, it has been feasible to change many aspects of sleep management across a breadth of inpatient units in a large NHS trust.

Let's get moving! Improving physical activity amongst rehabilitation patients; a quality improvement project

Ruth Rowland ${ }^{\star}$, Laura Somerville, Sarah Dorman and Mark Finnerty

Downshire Hospital

${ }^{*}$ Corresponding author.

doi: 10.1192/bjo.2021.576 
Aims. This Quality Improvement Project aimed to improve physical activity amongst patients in a 16-bedded, low secure unit in the Downshire Hospital, Northern Ireland. We introduced an exercise programme with the aim of increasing minutes of physical activity per week. Secondary outcome measures were weight, mood and energy levels.

This project took place in the context of COVID-19 restrictions having reduced opportunities for off-ward activity and staff noting subsequent deconditioning and weight gain amongst the patient cohort. Cohort consisted largely of patients with a severe mental illness, many of whom had physical health co-mobridities.

Method. This project included all patients in the 16-bedded unit.

Baseline data were collected prior to programme introduction, including weekly activity levels and weights. A questionnaire explored patient confidence and attitude towards physical activity.

Focus groups were held with patients and staff in order to identify how best to introduce the programme, discuss content, and identify potential barriers.

We introduced an eight-week programme of weekly, thirtyminute, mixed ability exercise sessions. These were led collectively by the multi-disciplinary team. Patients actively participated in programme design; choosing session soundtracks and contributing to content planning.

Likert scales were used to measure self-report mood and energy levels pre- and post-session. Staff engaged in a weekly post session de-brief, where challenges were identified and solutions suggested. Weekly qualitative feedback was sought from participants. The sessions were thus developed and adapted according to patient and staff feedback over the programme's course.

Following the 8-week programme, activity levels and weight were re-measured and compared to baseline. Pre-programme questionnaires were also repeated.

Result. Patients reported increased enjoyment and confidence engaging in physical activity, as well as improved overall self- confidence and a sense of pride and ownership of the sessions.

Staff reported a more cohesive team environment, greater sense of work-place fulfilment and improved therapeutic relationships.

Comparing pre and post session ten-point-Likert scales showed a $153 \%$ mean increase in self-rated energy levels and a $98 \%$ mean increase in self-rated mood. This reflected a mean score increase of 3.8 in both.

Minutes of physical activity per week increased for all session participants, although remained below national guidance.

Weight reduction did not occur.

Conclusion. Exercise benefits not only physical health, but also emotional and psychological well-being. This project demonstrates how introduction of a weekly ward-based exercise class can offer this as well as improving working environment, team cohesion and therapeutic relationships. Weight reduction may be observed in the longer term.

\section{Survey to evaluate care of complex clients in residential setting \\ Rosa Sadraei ${ }^{1 \star}$, Puru Pathy ${ }^{2}$ and Michael Collins ${ }^{1}$ \\ ${ }^{1}$ Nottinghamshire Healthcare NHS Foundation Trust and \\ ${ }^{2}$ Nottinghamshie Healthcare NHS Trust \\ ${ }^{\star}$ Corresponding author.}

doi: 10.1192/bjo.2021.577

Aims. Delivering a new efficient assessment and shorter term secondary mental Health intervention service for individual sectors
Background. In November 2015, there was a transition to services with the focus on delivering more efficient service to clients

Previously we had been a combined sector Service. This transition, a reduction in resources and a move away from delivering care Through specialist mental health teams created from the national service framework - such as Assertive outreach, early intervention in psychosis and community rehabilitation - to a more Streamlined generic service, catering for these differing groups of people using a "Pathways Model" approach

Result. Across the two sectors we had 47 clients on CPA Pathway living in 24 hour residential Settings who all had a current care coordinator.

These 47 clients represented the workload currently of 2.8 FTE Band 6 care coordinators.

There were at Origin, 13 Residential/Nursing/Secure 24 Hour care providers, where clients were residing.

However of these $90 \%$ of residents lived in one of 5 settings, 3 settings in Ashfield and 2 in Mansfield.

Over $50 \%$ of individuals residing did not have existing connections with Mansfield or Ashfield before being placed into the area.

18 Clients (\%38) were under section of the mental health act and 1 client (\%2) was on a life-Licence from criminal justice.

Conclusion. Transfer of CPA Care Coordination Protocol

To send paper referral to our Single Point of Access Meeting at the listed address at the earliest point relocation/placement is confirmed.Formal handover meeting for care will be coordinated, not sooner than 3 months after the placement commences. It will be expected that services currently involved in provision of service continue to hold care responsibility in the interim period.

As we move to a paperless environment, provision of electronic documentation such has previous CPA documents, Risk assessments, social circumstance reports \& Discharge summaries, would be greatly appreciated

\section{Patient \& staff perceptions of animal-assisted therapy in psychiatric rehabilitation}

Laura Sallette $^{1 \star}$, David King ${ }^{2}$, Sian Cowton-Williams ${ }^{1}$ and Rajesh Mohan ${ }^{1}$

${ }^{1}$ Heather Close Rehabilitation Unit, South London and Maudsley NHS Foundation trust and ${ }^{2}$ King's college london

${ }^{\star}$ Corresponding author.

doi: 10.1192/bjo.2021.578

Aims. To study patients' subjective experiences of having access to a therapy dog and to assess the staff perception of the impact of pet therapy. Hypothesis: pet therapy services are acceptable for rehabilitation patients.

Background. Animal-assisted therapy (AAT) is the supervised use of an animal in a therapeutic setting to help in the treatment of physical or psychological disorders in humans. The use of dogs in the context of AAT - 'dog therapy' (DT) has been piloted in the context of stroke rehabilitation; schizophrenia in elderly patients; depression, loneliness and anxiety in elderly patients; Alzheimer's disease; symptom reduction in PTSD; cognitive impairment; and dementia. The impact of pet therapy in long term psychosis care has not been adequately assessed.

Method. This feasibility pilot study used questionnaires to assess patient $(n=12)$ and staff $(n=10)$ perceptions of dog therapy in an in-patient psychiatric rehabilitation setting. 24 patients on a rehabilitation ward with complex psychosis were offered the opportunity to interact with 'Nugget,' a corgi trained in the 